\title{
Reply to Stefano Miceli Sopo
}

\author{
Susanne Halken ${ }^{1}$, Muraro Antonella ${ }^{2}$, Graham Roberts ${ }^{3}$, and Debra de Silva ${ }^{4}$ \\ ${ }^{1}$ Odense University Hospital \\ ${ }^{2}$ University Hospital of Padua \\ ${ }^{3}$ University of Southampton \\ ${ }^{4}$ The Evidence Centre
}

February 14, 2022

\section{To the Editor}

\section{Reply to Stefano Miceli Sopo}

First of all we want to thank for giving us an opportunity to reply to this correspondence (1), acknowledging the correspondence authors important point about the recommendation of introducing well-cooked, but not raw or uncooked pasteurized hen's egg as part of complementary feeding.

We agree that the evidence-base is sparse, with just two trials about cooked egg contributing to the low to moderate certainty evidence in our review (2) and guideline (3). These studies were in different populations $(4,5)$. We highlighted these points in the guideline, and the subgroup analysis from the Perkin study (6) was used only as supporting material, and for estimating the amount of egg that could be used. It is correct that the Natsume study included infant at higher risk due to eczema, but that was the case for both groups, and outcome was assessed by controlled challenges as described in the systematic review (2).

As set out in the guideline, the process took into account expert insight weighing up benefits and harms, costs, feasibility, standard practice and patient preferences, in addition to published evidence. Weighing up all of these factors, the task force decided that the potential benefits outweighed potential harms in the case of well-cooked egg. One relatively large study found a $29 \%$ absolute decrease in the proportion of high risk infants with egg allergy at 1 year when very small amounts of egg were introduced (RR $0.22,95 \%$ CI 0.08 to 0.54) (4). And two trials found no adverse effects $(4,5)$. It is likely feasible for many families to introduce well-cooked egg as part of complementary feeding, including in baked goods. The potential benefits do not outweigh the harms for uncooked egg, so the task force did not suggest trying this approach.

The task force included representatives from many countries and specialties, and followed a robust process when reviewing evidence and debating potential recommendations. As the correspondence authors note, this recommendation is in line with other key guidelines. Whilst the correspondence authors may not agree with specific recommendations, the process used to debate and vote on them was systematic and took into account perspectives from across the world, including those from organisations representing patients and their families. Furthermore, a public consultation process sought feedback prior to publication, which further reinforced consensus about this recommendation.

As is the case with all guidelines, the EAACI food allergy prevention guideline provides suggestions for clinicians to consider, alongside the needs of individual patients and local contexts and customs. The guideline is not prescriptive and does not override clinical judgement ad individual circumstances. Given the lack of likely harm, the convenience of this approach and best available evidence to date, the task force stands by its suggestion that clinicians in countries where egg allergy is an issue discuss with families the potential and desire to introduce small amounts of well-cooked egg into the infant diet when appropriate 
as part of complementary feeding. This need not be from the beginning of complementary therapy and the amounts may be very small.

The guideline suggests half of a well-cooked, small egg twice a week, which may be in the form of a hardboiled egg, well-cooked egg pasta, bread or baked goods, for example (p. 850). There is no evidence of significant harm, and it is likely that infants in many parts of the world may be exposed to egg in their diet anyway. There is no need to avoid this to prevent egg allergy, and in the opinion of the EAACI task force, introducing it may have benefits.

Susanne Halken, Professor ${ }^{a}$, Professor

Antonella Maria Muraro b, Professor

Graham Roberts ${ }^{\mathrm{c}}$, Professor

Debra de Silva ${ }^{\mathrm{d}}$, Professor

On behalf of On behalf of the EAACI Prevention Guideline Task Force

a Hans Christian Andersen Children's Hospital, Odense University Hospital, Odense, Denmark

${ }^{\mathrm{b}}$ Department of Women and Child Health, Food Allergy Referral Centre Veneto Region, Padua University Hospital, Padua, Italy

c Clinical and Experimental Sciences and Human Development in Health, Faculty of Medicine, University of Southampton, Southampton, UK. NIHR Southampton Biomedical Research Centre, University Hospital Southampton NHS Foundation Trust, Southampton, UK. The David Hide Asthma and Allergy Research Centre, St Mary's Hospital, Newport, UK

dThe Evidence Centre Ltd, London, UK

References

1. Stefano Miceli Sopo, Dario Sinatti, Francesco Mastellone, Giulia Bersani, Mariannita Gelsomino. Comment on Halken et al. Pediatr Allergy Immunol . 2022

2. de Silva D, Halken S, Singh C, et al. Preventing food allergy in infancy and childhood: systematic review of randomised controlled trials. Pediatr Allergy Immunol . 2020;31(7):813-826

3. Halken S, Muraro A, de Silva D, et al. EAACI guideline: Preventing the development of food allergy in infants and young children (2020 update). Pediatr Allergy Immunol. 2021;32(5):843-858.

4. Natsume O, Kabashima S, Nakazato J, et al. Two-step egg introduction for prevention of egg allergy in high-risk infants with eczema (PETIT): a randomised, double-blind, placebo-controlled trial. Lancet. 92 2017;389(10066):276-286.

5. Perkin MR, Logan K, Tseng A, et al. Randomized trial of introduction of allergenic foods in breast-fed infants. N Engl J Med. 2016;374(18):1733-1743.

6. Perkin MR, Logan K, Bahnson HT, et al. Efficacy of the enquiring About Tolerance (EAT) study among infants at high risk of developing food allergy. J Allergy Clin Immunol. 2019;144(6):1606-1614. 